\title{
BEYOND POSSIO EQUATION: THE LEGACY OF CAMILLO POSSIO TO FLIGHT DYNAMICS AND HYDRODYNAMICS
}

\author{
G. Avanzini ${ }^{1}$ and A. De Santis, ${ }^{2}$ \\ ${ }^{1}$ Politecnico di Torino, Dipartimento di Ingegneria Aeronautica e Spaziale, Turin, \\ Italy, giulio.avanzini@polito.it ${ }^{2}$ Università degli Studi di Roma "La Sapienza", Dipartimento \\ diInformatica e Sistemistica, desantis@dis.uniromal.it
}

\begin{abstract}
A review of the papers written by Camillo Possio on Flight Dynamics and Hydrodynamics is presented. The scope of the note is to underline how the versatile young researcher succeeded in delivering interesting contributions to the engineering sciences that go beyond the renown equation that bears his name.
\end{abstract}

keywords: aircraft stability derivatives, free surface, marine propeller

\section{Introduction}

The works of Camillo Possio on unsteady aerodynamics [1]-[7] have deeply influenced the studies in the field of aeroelasticity up to the present days, as demonstrated by some recent works that exploited his derivations [8]-[10]. For this reason the related papers are considered as his most important contributions to the engineering disciplines. As a matter of fact his scientific production was extremely prolific and diverse in spite of his untimely death under the last bombing that hit his native city, Turin, at the end of the Second World War, on April 5th, 1945.

Beyond his seminal contribution to unsteady aerodynamics, Possio's works ranged from the analysis of fluid motion $[11,12]$ to studies on physical properties of fluids [13], from flight mechanics [14]-[16] and experimental fluid dynamics [17] to free surface effects on the flow field generated by distributions of singularities [18]-[20]. These works deserve consideration not only because they reveal the versatility of his mind in applying a rigorous mathematical approach for describing physical phenomena, always preserving a deep practical understanding of the underlying physical system, but also because they are undoubtedly as interesting as the most renown ones. The present note will focus on five papers that represent the legacy of Possio to the fields of flight dynamics ([14] and [15]) and hydrodynamics ([18]-[20]), discussing

Please use the following format when citing this chapter:

Author(s) [insert Last name, First-name initial(s)], 2006, in IFIP International Federation for Information Processing, Volume 199, System Modeling and Optimization, eds. Ceragioli F., Dontchev A., Furuta H., Marti K., Pandolfi L., (Boston: Springer), pp. [insert page numbers]. 
their significance in the framework of the current studies of the time on the two subjects.

In Refs. [14] and [15], both written in 1938, Possio exploited his experience in managing basic models of oscillating lifting surfaces in order to derive some analytical results aimed at evaluating aircraft stability derivatives, that is the expressions of the perturbation terms of aerodynamic forces and moments that are required in the linearization of rigid aircraft equations of motion. The last part of his scientific activity was focused on the analysis of free-surface effects. In particular, between 1941 and 1943 he wrote a sequence of three papers that were devoted to the analysis of steady motion of heavy fluids under the influence of a distribution of singularities [18], the interaction of a vortex in steady motion with a free-surface [19], and to the estimate of marine propeller efficiency as a function of its depth [20], pushing further the analysis of the problem that had been given approximate solutions by other scientists of the time.

Without neglecting the originality and potential importance of the works that will not be discussed in the present note, the legacy of Camillo Possio to flight dynamics and hydrodynamics represents the major contribution (beyond Possio equation) that the young scientist could develop into a complete research, obtaining results that represented an advance in engineering knowledge at the time the papers were written, while preserving also nowadays a significant interest for the aerospace community.

\section{Possio and aircraft stability derivatives}

On January 1938 Camillo Possio published on the Commentationes Pontificia Academia Scientiarum (Proceedings of the Pontifical Academy of Sciencies) a work titled "On the Determination of Aerodynamic Coefficients for Aircraft Stability Analysis" [14]. The importance of this work can be easily understood when one considers that the formulation of rigid aircraft equations of motion had already reached full maturity for a long time in 1938, the seminal book by Bryan [21] dating back to 1911, but the evaluation of the so called dynamic stability derivatives was still an open problem.

The approach proposed by Bryan for the analysis of aircraft stability is based on the derivation of the equations of motion from first principles, the determination of aircraft equilibria (trimmed flight) and the linearization of the equations of motion in the neighborhood of the considered trim condition. In Bryan's theory, aerodynamic forces and moments are expanded in linear form as a function of aircraft state and control variables, under some reasonable simplifying assumptions.

After writing the linear model, the main problem for a meaningful aircraft stability analysis is to provide reasonable estimates of aircraft stability derivatives, that is, the partial derivatives of aerodynamic forces and moments with 
respect to state and control variables, divided by a mass parameter (the mass for force equations or the relevant moment of inertia for each one of the three attitude equations). Stability derivatives may change significantly, depending on the considered trim condition and aircraft configuration. The determination of the so called static stability derivatives (that is, stability derivatives made with respect to aerodynamic angles or velocity components) can be performed with different degrees of accuracy, but a reasonable estimate is not too a difficult task. On the converse, the evaluation of rotary and dynamic derivatives, that is stability derivatives with respect to angular velocity components and time derivatives of aerodynamic angles, is never trivial. In his earliest work on aircraft stability (that was written with W.E. Williams in 1903 before Wright brothers' first powered flight, and published on January 1904 only three weeks after the Flyer took-off!), Bryan simply neglected the terms depending on the rate of change of incidence and sideslip angles [22]. Flight experience and more accurate mathematical derivations rapidly demonstrated that unsteady downwash effects on the horizontal tail were sizable and cannot be neglected.

Unfortunately, dynamic derivatives escape also direct experimental evaluation, not only because of scale and wind-tunnel effects (the latter analyzed by Possio in [17]) but also because forced oscillation experiments cannot determine separately the contributions of angular velocity and rate of variation of aerodynamic angles to force and moment components.

As for evaluation of dynamic stability derivatives from theoretical aerodynamic results, Possio himself underlined how the effects of small amplitude oscillations on lift distribution cannot be described by a two-dimensional representation, that, together with other limitations, would make it impossible to evaluate stability derivatives with respect to roll angular velocity, nor by a simple strip theory, even under the assumption of a low frequency parameter $\omega=\Omega b /\left(2 V_{0}\right), \Omega$ being the frequency of the oscillation, $b$ the wing span and $V_{0}$ the trim velocity [14]. Exploiting his analysis of the behavior of oscillating lifting surfaces, Possio derived a rigorous description of the vorticity and pressure distributions over a lifting surface in a harmonic oscillation, under the assumptions of small incidence and oscillation amplitude and large wing aspect ratio $\lambda$.

His derivation for a wing like that shown in Fig. 1 was based on Prandtl's acceleration potential approach [23]. The total derivative of the velocity $\mathbf{V}$ can be expressed as

$$
\frac{d \mathbf{V}}{d t}=\operatorname{grad} \varphi
$$

where the acceleration potential $\varphi$ satisfies Laplace equation $\nabla^{2} \varphi=0$. Writing Eq. (1) in linear, nondimensional form (that is assuming small perturbation of a uniform flow $\mathbf{V}_{0}$ of unity modulus), the vertical component $v$ of the velocity 


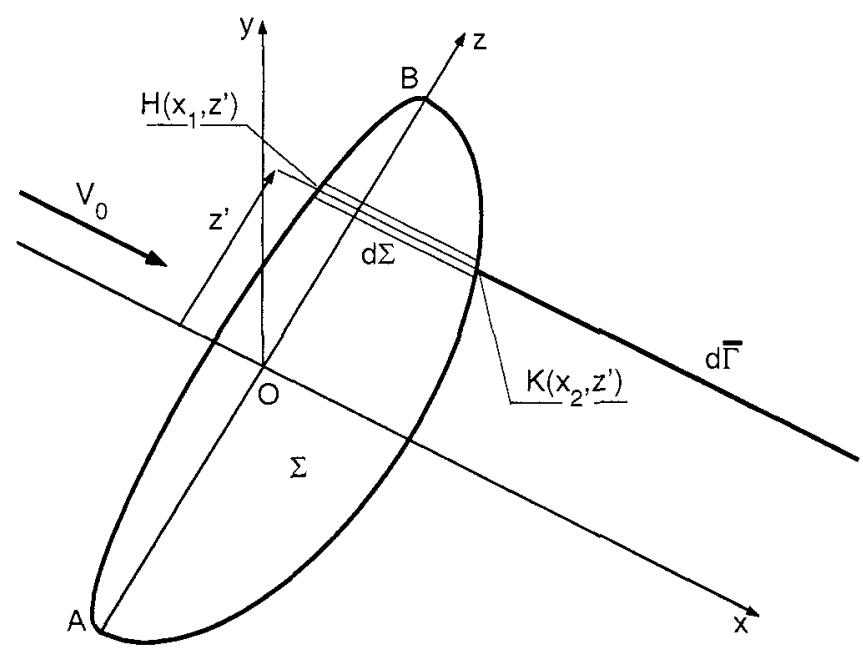

Figure 1. Elliptic wing in a uniform current [14]

field must satisfy the equation

$$
\frac{\partial \varphi}{\partial y}=\frac{\partial v}{\partial x}+\frac{\partial v}{\partial t}
$$

Taking into account that $v \rightarrow 0$ for $x \rightarrow-\infty$, it is possible to integrate Eq. (2), so that

$$
v(x, y, z, t)=\int_{-\infty}^{x} \frac{\partial \varphi}{\partial y}(\xi, y, z, t+\xi-x) d \xi
$$

The restriction of $v$ over the surface $\Sigma$ of the oscillating wing must satisfy the flow tangency condition on $\Sigma$, that is $v(x, z)=\partial \eta / \partial t-\alpha$, where $\eta$ is the vertical displacement of the point $(x, z) \in \Sigma$ and $\alpha$ the wing incidence. For a harmonic oscillation all the terms can be expressed in complex form as $\varphi=\bar{\varphi} e^{i \omega t}$ and $v=\bar{v} e^{i \omega t}$, where $i$ is the imaginary unit and $\bar{\varphi}$ and $\bar{v}$ are complex functions of $x, y$, and $z$. The restriction of $\bar{v}$ over $\Sigma$ can thus be written as

$$
\bar{v}(x, z)=\int_{-\infty}^{x} \frac{\partial \bar{\varphi}}{\partial y}(\xi, z) e^{i \omega(\xi-x)} d \xi
$$

Noting that for incompressible fluids no time derivatives of $\varphi$ are present in the Laplace equation governing acceleration potential, it is possible to state that $\bar{\varphi}$ is the acceleration potential of a steady flow field $C_{p}$ with asymptotic velocity $V_{0}$ around a lifting surface with the same shape of $\Sigma$. Indicating the horizontal and vertical velocity components of the steady flow field $C_{p}$ with $1+u^{\prime}$ and $w$, respectively, and applying Bernouilli's theorem for small 
perturbations $u^{\prime}, w \ll 1$, it is $\bar{\varphi}=u^{\prime}$. It is also $\partial \bar{\varphi} / \partial y=\partial w / \partial x$, because $C_{p}$ is irrotational. By substituting $w$ in Eq. (3) and integrating by parts, one gets

$$
\bar{v}(x, z)=w(x, z)-i \omega \int_{-\infty}^{x} w(\xi, z) e^{i \omega(\xi-x)} d \xi
$$

The flow field $C_{p}$ can be generated by a (steady) complex vorticity distribution $\bar{\gamma}(x, z)$ over $\Sigma$, that Possio determined following Prandtl theory for steady motion of finite wings, expressing $\bar{\gamma}$ in terms of Glaurt's trigonometric series. The details of the procedure are here omitted for the sake of brevity, but can be found on Possio's paper [14]. It must be observed that, as explicitly underlined by Possio himself, the flow field $C_{p}$ and its vertical component $w$ bear no physical meaning. In particular, although $w(x, z)$ can be decomposed into the sum of two contributions $w_{1}$ and $w_{2}$ related to the circulation distribution in $C_{p}$ on the wing and downstream of it, respectively, the two corresponding terms $\bar{v}_{1}$ and $\bar{v}_{2}$ obtained by substituting $w_{1}$ and $w_{2}$ in Eq. (4) are not related to the unsteady distribution of vorticity over and downstream of the wing in the actual unsteady flow field.

In the final part of the paper Possio derived some simplified relations for the particular case of an elliptic wing. He also demonstrated that the approximation of his prediction for the unsteady case has the same accuracy of Prandtl's model for the steady case and developed a simple example for an isolated wing which undergoes a vertical harmonic oscillation. He anticipated that a complete set of results on aerodynamic forces and moments generated by roll, pitch, yaw and heave oscillations of a complete aircraft configuration would have been discussed in a subsequent note. On December 1938 this note was published by the italian journal L'Aerotecnica under the title "Determination of Aerodynamic Actions Due to Small Aircraft Oscillations" [15]. Figure 2 shows some of Possio's original plots, namely Figs. 3 through 9 taken from Ref. [15].

It should be noted that Possio presented his results adopting the italian notation in use at his time, where $C_{p}$ represents the lift coefficient, from the word portanza, and $C_{m r}$ is the roll moment coefficient, where $m r$ stands for momento di rollio. In the paper, $C_{r}$ indicates the drag coefficient (resistenza), $C_{d}$ the side-force coefficient (deriva) while $C_{m b}$ and $C_{m i}$ indicate the pitch and yaw moments (beccheggio and imbardata), respectively.

In the last paragraph of the paper Possio carried out a numerical example for evaluating the differences with respect to what he called "the usual approximate methods" that neglect part of the unsteady aerodynamic effects, rigorously taken into account by his approach. While the terms in phase with the variation of the angle of attack where shown to be predicted well even by the approximate methods, the terms in quadrature with $\alpha$ were demonstrated to be significantly different, with variations that ranged from $28 \%$ for the pitch moment stability 

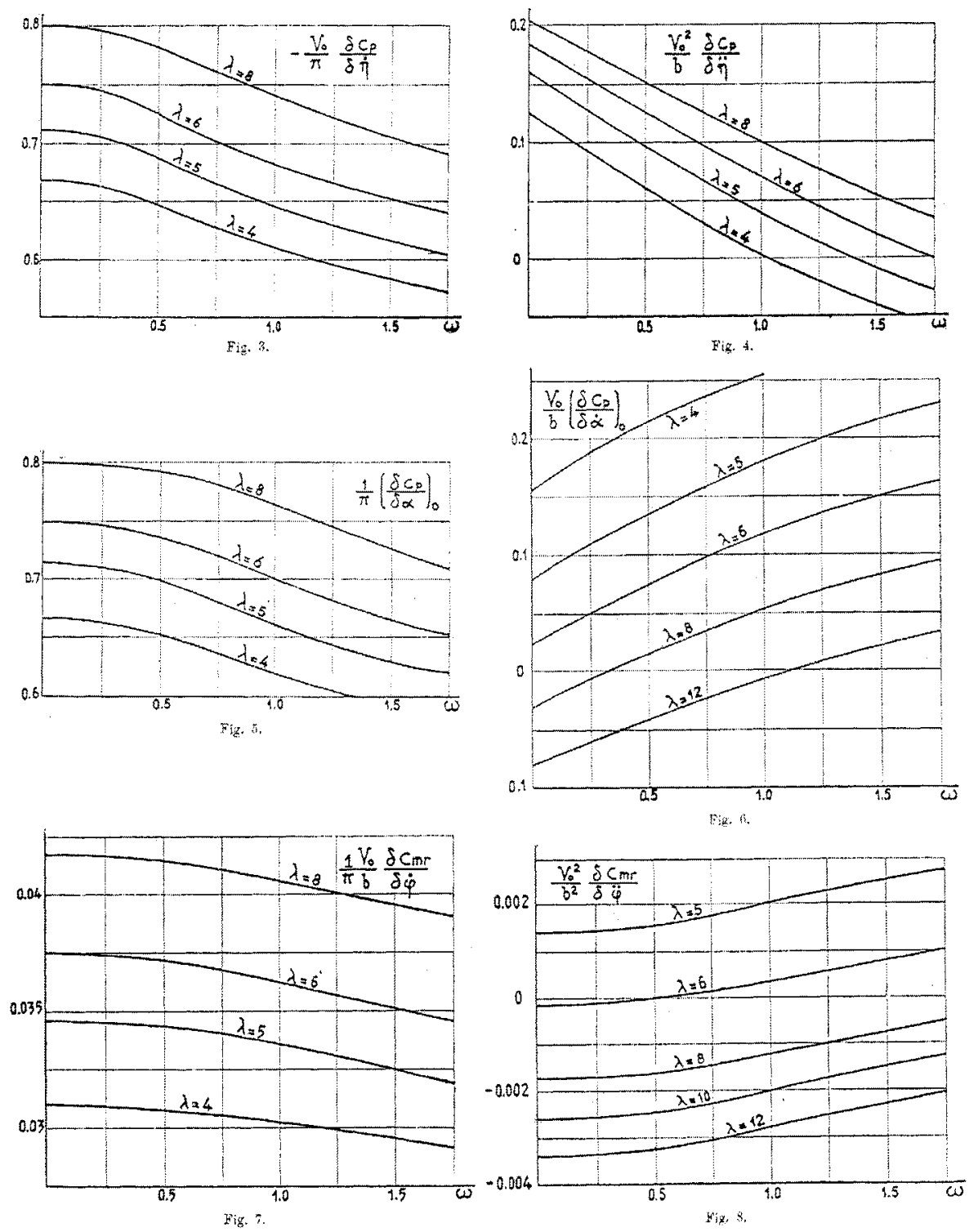

Figure 2. Lift coefficient $C_{p}$ in plunge (Figs. 3 and 4 ) and pitch motion (Figs. 5 and 6), and roll coefficient $C_{m r}$ for roll oscillations (Figs. 7 and 8) from Ref. [15] 
derivative with respect to $\dot{\alpha}$ up to $200 \%$ for the lift derivative with respect to $\alpha$ [15].

\section{Possio's analysis of free-surface effects}

In 1941 Possio published his first work on free surface effects on the Annali di Matematica Pura e Applicata (Annals of Pure and Applied Mathematics) [18], analyzing the steady velocity field created by a distribution of singularities on the free surface of a heavy fluid.

Lamb and Havelock demonstrated that under the usual hypotheses of perfect fluid and infinitesimal perturbation an infinite set of solutions is analytically plausible for this problem [24],[25]. Indicating with $V$ and $V_{0}$ the local and the asymptotic velocity, respectively, Rayleigh proposed a method to define a well-posed problem by assuming that an elementary viscous-like force per unit mass in the form $-\mu\left(V-V_{0}\right)$ acts on the fluid, where $\mu$ is a positive parameter. The flow field for a perfect fluid was thus obtained in the limit as $\mu \rightarrow 0$.

Possio provided an alternative formulation based on a more rigorous model. While retaining the assumption of perfect fluid, he considered the stationary motion as the limit of an unsteady one, started with the fluid at rest when a perturbation created by the singularities is suddenly applied at $t=0$. The general solution can be expressed in terms of velocity potential as $\Phi(x, y, z, t)=$ $\Phi_{i}(x, y, z)+\Phi_{a}(x, y, z, t)$, where $\Phi_{i}$ is the potential determined by the singularities distribution and its value is suddenly assumed at $t=0$, while $\Phi_{a}$ is an additive potential satisfying the Laplace equation $\nabla^{2} \Phi_{a}=0$, with continuous first derivatives in the half-space $z \geq 0$ and vanishing at infinity except in the downstream direction. The formulation of the unsteady problem is well posed and it admits a unique solution. If the perturbation is created by a distribution of sources or doublets, the solution is given by

$$
\begin{aligned}
\Phi_{i}(x, y, z) & =\int_{-\pi}^{\pi} d \theta \int_{0}^{\infty} F(m, \theta) e^{m k z+i m k \omega} d m \\
\Phi_{a}(x, y, z, t) & =\int_{-\pi}^{\pi} d \theta \int_{0}^{\infty} f(m, \theta, t) e^{m k z+i m k \omega} d m
\end{aligned}
$$

with $k=g / V_{0}^{2}$ and $\omega=x \cos (\theta)+y \sin (\theta) . F(m, \theta)$ is a continuous bounded function, with continuous bounded first derivatives in the set $m \geq 0$ and $-\pi \leq$ $\theta \leq \pi$, such that $F \rightarrow 0$ as $e^{-m k l}$ if $m \rightarrow \infty$. Finally, $f$ is an arbitrary function such that the integral is defined in $z \geq 0$ and differentiation under the integral sign is also defined.

Possio demonstrated that the stationary additional potential $\Phi_{a}^{*}$ obtained as $\lim _{t \rightarrow \infty} \Phi_{a}(x, y, z, t)$ coincides with the expression obtained by Rayleigh, and satisfies the limit condition imposed on the potential and its first derivatives at infinity. He also extended his approach to a more general situation where the 
perturbation is given by a pressure discontinuity, so as to include the flow field generated by a propeller or an airfoil.

This investigation was naturally developed into the study of the potential that describe the velocity field generated by a vortex with circuitation $\Gamma$ in uniform motion at a depth $h$ from a free surface [19]. In this case the potential can be expressed as

$$
\Phi(x, y)=V_{0} x+\frac{\Gamma}{2 \pi} \tan ^{-1}\left(\frac{y-h}{x}\right)+\Phi_{a}(x, y)
$$

where $\Phi_{a}$ is the additional potential required to satisfy the boundary condition at the free surface, that is, pressure $p$ in the fluid at the surface is equal to the external pressure $p_{a}$ in the space over the surface. As usual, the potential function $\Phi_{a}$ must satisfy the Laplace equation $\nabla^{2} \Phi_{a}=0$ in the half-space occupied by the fluid, the first derivatives vanishing upstream of the vortex, so that a well-posed problem is obtained.

Possio found the expression of $\Phi_{a}$ and investigated its numerical computation. The theory was then applied to the problem of the uniform motion of an airfoil of chord $\ell$ for small values of the ratio $\varepsilon=\ell / h$. The resulting lift and drag coefficients were expressed as

$$
c_{L}=\frac{\pi \alpha}{1+\pi \beta e^{-2 \beta} \varepsilon}, \quad c_{D}=\beta e^{-2 \beta} \varepsilon c_{L}^{2}
$$

with $\beta=g h / V_{0}^{2}$. The aerodynamic coefficients were similar to those obtained by Prandtl for the small $\varepsilon$ case, expressed in terms of a virtual aspect ratio $\lambda=2 e^{2 \beta} / \pi \beta \varepsilon$. Possio determined $\lambda$ for arbitrary values of $\varepsilon$, although his analysis was limited to the flat plate. In this case the virtual aspect ratio is given by

$$
\frac{\lambda=2 F^{2} e^{2 /\left(\varepsilon F^{2}\right)}}{\pi\left\{J_{0}^{2}\left[1 /\left(2 F^{2}\right)\right]+J_{1}^{2}\left[1 /\left(2 F^{2}\right)\right]\right\}}
$$

where $J_{0}()$ and $J_{1}()$ are Bessel functions and $F=V_{0} / \sqrt{g \ell}$ is the Froud number. The diagram of Fig. 3 represent the behaviour of $1 / \lambda$ as function of $F$ for different values of $\varepsilon$.

In Ref. [20] Possio investigated free surface effects on propeller efficiency. This problem had been already addressed by Dickmann [26], under the assumption that the field external to the wake created by the propeller can be represented by a distribution of sinks over the propeller disc, thus exploiting the results of Havelock on wave resistance generated by a given singularities distribution [25].

Possio provided a more accurate computation of wave resistance and extended the investigation to the case of more than one propeller. Letting $h$ be the depth of the propeller disc center, measured along the $z$ axis oriented downward, and $\varepsilon=r / h$ the ratio between the disc radius and the depth, Possio 


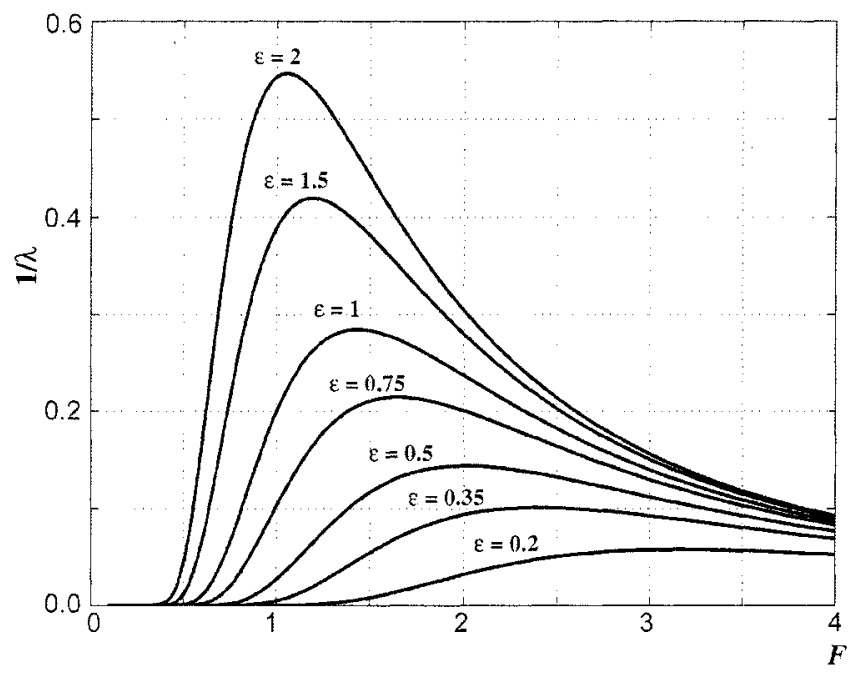

Figure 3. Reciprocal of virtual aspect ratio as a function of Froud number.

represented the wave resistance generated by a uniform distribution of sinks on the propeller disc $\sigma$ as

$$
R_{0}=8 \pi \rho\left(\frac{\pi m r^{2}}{h}\right)^{2} f, \quad f=2 \sum_{0}^{\infty} a_{n} \varepsilon^{2 n} I_{n}(\beta)
$$

where $\beta=g h / V_{0}^{2}$ and

$$
I_{n}(x)=x^{2 n+2} e^{-x} \int_{0}^{\infty} e^{-x \cosh (2 u)} \cosh ^{2 n+2}(u) d u
$$

Dickmann results refers to the case of a sink distribution concentrated in the disc center with $\varepsilon=0$, that is just the first term in the series expansion of Eq. 10 . On the converse, Possio computed $f$ for different values of the ratio $\varepsilon$ and of the Froud number.

He also evaluated propeller efficiency loss due to wave motion induced on the free surface. Denoting with $u_{m}^{*}$ the average velocity increment at the propeller, with $P$ and $T$ the propeller power consumption and thrust, respectively, the actual propeller efficiency is $\eta_{e}=T V_{0} / P$, while the efficiency in an infinite fluid is $\bar{\eta}=T\left(V_{0}+u_{m}^{*}\right) / P$. By taking into account that $u_{m}^{*} / V_{0} \ll 1$, it is possible to write

$$
\frac{\bar{\eta}-\eta_{e}}{\bar{\eta}}=\varepsilon^{2} f \frac{u_{d}}{V_{0}}
$$

where $u_{d}$ denotes the velocity increment at the propeller disc. 


\section{Conclusions}

Camillo Possio demonstrated with his extremely diverse scientific activity an unusual capability of handling complex mathematical models while preserving a deep physical insight on the underlying engineering problem. This note was aimed at presenting his works on flight dynamics and hydrodynamics subjects, the only fields where fate allowed the young scientist to deliver an articulate contribution beyond the state of the art of his time and beyond the seminal equation that bears his name.

\section{References}

[1] C. Possio. L'azione aerodinamica sul profilo oscillante alle velocità ultrasonore. Acta Pontificia Academia Scientiarum. 1(11):93-106, 1937.

[2] C. Possio. L'azione aerodinamica sul profilo oscillante in un fluido compressibile a velocità iposonora. L'Aerotecnica. 18(4):441-458, 1938.

[3] C. Possio. L'azione aerodinamica su una superficie portante in moto oscillatorio. Atti Reale Acc. Lincei. 26:194-200, 1938.

[4] C. Possio. Sul moto non stazionario di una superficie portante. Atti Reale Acc. delle Scienze - Parte Fisica. 74:285-299, 1939.

[5] C. Possio. L'azione aerodinamica su di una superficie portante in moto vario. Atti Reale Acc. delle Scienze - Parte Fisica. 74:537-557, 1939.

[6] C. Possio. Sul problema del moto discontinuo di un'ala - Nota la (Moto Piano). L'Aerotecnica. XX(9):655-681, 1940.

[7] C. Possio. Sul problema del moto discontinuo di un'ala - Nota 2a (Ala finita). L'Aerotecnica. XXI(3):205-230, 1941.

[8] G. Chiocchia, S. Prößdorf, D. Tordella. The lifting line equation for a curved wing in oscillatory motion. ZAMM, 77(4):295-315, 1997.

[9] J. Lin, K.W. Iliff. Aerodynamic Lift and Moment Calculations Using a Closed Form Solution of the Possio Equation. Nasa/TM-2000-209019, 2000.

[10] A.V. Balakrishnan. Possio Integral Equation of Aeroelasticity Theory. J. Aerosp. Engrg. 16(4):139-154, 2003.

[11] C. Possio. Sul moto rotazionale dei gas. Atti Reale Acc. Lincei. 25:455-461, 1937.

[12] C. Possio. Sul moto non stazionario di un fluido compressibile. Rendiconti Reale Acc. Lincei:481-487, 1939.

[13] C. Possio. L'influenza della viscosità e della conducibilità termica sulla propagazione del suono. Atti Reale Acc. delle Scienze - Parte Fisica. 77:274-293, 1942.

[14] C. Possio. Sulla determinazione dei coefficienti aerodinamici che interessano la stabilità del velivolo. Commentationes Pontificia Academia Scientiarum. III(6):141-169, 1938.

[15] C. Possio. Determinazione dell'azione aerodinamica corrispondente alle piccole oscillazioni del velivolo. L'Aerotecnica. XVIII(12):1323-1351, 1938.

[16] C. Possio. Sullo sparo di fianco da bordo di un aereo. Atti Reale Acc. delle Scienze-Parte Fisica. 74:276-285, 1939.

[17] C. Possio. L'interferenza della galleria aerodinamica nel caso di moto non stazionario. VI Convegno Nazionale di Aerotecnica,306-320, Rome, June 1940. 
[18] C. Possio. Sulla teoria del moto stazionario di un fluido pesante con superficie libera. Annali di Mat. Pura e Appl.. 20:313-329, 1941.

[19] C. Possio. Campo di velocità creato da un vortice in un fluido pesante a superficie libera in moto uniforme. Atti Reale Acc. Delle Scienze - Parte Fisica. 76:365-388, 1941.

[20] C. Possio. L'influenza della superficie libera sul funzionamento dell'elica marina. Sistemi di Propulsione e Navigazione aerea e marittima, 334-352, Torino, Acc. Delle Scienze, 1943.

[21] G.H. Bryan. Stability in Aviation. MacMillan, London, 1911.

[22] G.H. Bryan, W.E. Williams. The Longitudinal Stability of Aerial Gliders. Proc. Royal Soc. London Ser. A, 73(489): 100-116, 1904.

[23] L. Prandtl. Beitrag zur Theorie der tragenden Flache. ZAMM. 16(6):360-361, 1936.

[24] H. Lamb. Hydrodynamics, $6^{\text {th }}$ ed. Cambridge University Press, Cambridge, 1932. Chapt. IX.

[25] T.H. Havelock. Wave resistance. Proc. Royal Soc. London. Ser. A, 118:24-33, 1928.

[26] H.E. Dickmann. Schiffskörpersog, Wellenwiderstand des Propellers und Wechselwirkung mit Schiffswellen. Ing-Arch. 9, 1938. 